\title{
Soot Particle Deposition within Porous Structures Using a Method of Moments - Lattice Boltzmann Approach
}

\author{
Bernhard F.W. Gschaider, Claudia C. Honeger, and Christian E.P. Redl \\ Christian-Doppler-Laboratory for Applied Computational Thermofluiddynamics, \\ Mining University Leoben, \\ Franz-Josef-Strasse 18, 8700 Leoben, Austria
}

\begin{abstract}
This paper deals with the combination of two computational methods to simulate the flow of particle laden fluids through porous structures: the Lattice Boltzmann Method (LBM) which is a method to solve the Navier-Stokes equation in complex geometries and the Method of Moments (MoM) which describes the time evolution of nonhomogeneous particle distributions. The combination of these methods makes it possible to take phenomena into account that depend on particle size and size distribution of the transported material. It is also possible to simulate changes in the size distribution.
\end{abstract}

\section{Introduction}

The simulation of particle laden flows covers a large area of useful applications e.g. emissions of internal combustion engines, transport of pollutants in the ground-water, aerosols in the atmosphere.

Most of these applications involve the need to simulate a large number of particles with a non-uniform size distribution. This size distribution is described by a Probability Density Function (PDF). The shape of this PDF may change due to coagulation, nucleation or the breaking-up of particles.

A typical example for combined fluid-particle flow is the exhaust gas flow of combustion engines. A variety of problems arise within this field of application. One of these is the soot particle laden gas flow through particulate filter materials. The purpose of these simulations is to judge the efficiency of the filter material with respect to deposition of soot particles on the pore walls.

One difficulty with the simulation of flows inside porous materials is the complexity of the pore geometry. This makes it hard to generate a body fitted computational grid essential for traditional methods of flow simulation like the Finite Volume Method. Therefore a Lattice Boltzmann Method (LBM), which is especially well suited for complex geometries, is used. The computational domains for the LBM can be generated automatically from computer tomography or reconstructed using statistical correlations. 


\section{Lattice Boltzmann Method}

A standard incompressible formulation of the Lattice Boltzmann equation is used for simulating the fluid flow [1] [2].

The incompressible Lattice Boltzmann equation can be written as :

$$
f_{i}\left(t+1, \mathbf{x}+\mathbf{e}_{i}\right)=f_{i}(t, \mathbf{x})-\frac{1}{\tau}\left(f_{i}(t, \mathbf{x})-f_{i}^{e q}(t, \mathbf{x})\right)
$$

where the equilibrium distributions are given by

$$
f_{i}{ }^{e q}=t_{i}\left\{p+p_{0}\left(\frac{\mathbf{e}_{i \alpha} \mathbf{u}_{\alpha}}{c_{s}^{2}}+\frac{\mathbf{u}_{\alpha} \mathbf{u}_{\beta}}{2 c_{s}^{2}}\left(\frac{\mathbf{e}_{i \alpha} \mathbf{e}_{i \beta}}{c_{s}^{2}}-\delta_{\alpha \beta}\right)\right)\right\}+S_{i}
$$

In the above equations, $p_{0}$ is a reference pressure, $f_{i}$ are the distribution functions and $\mathbf{e}_{i}$ are the lattice vectors. $S_{i}$ is a volumetric source term which may result from the particle transport. The lattice dependent weighting factors for the D2Q9 model are $t_{0}=4 / 9$ for the rest particle distribution, $t_{1-4}=1 / 9$ for the cartesian directions and $t_{5-8}=1 / 36$ for the diagonal directed lattice vectors. The lattice speed of sound is $c_{s}=1 / \sqrt{3}$.

The hydrodynamic quantities can be obtained by computing the moments of the particle distribution functions :

$$
\begin{gathered}
p=\sum_{i} f_{i} \\
p_{0} \mathbf{u}=\sum_{i} f_{i} \mathbf{e}_{i}
\end{gathered}
$$

The relaxation parameter $\tau$ is related to the viscosity as follows:

$$
\tau=\frac{6 \nu+1}{2}
$$

Due to the tremendous computational overhead transport equations for species are not solved within the LBM framework but by other means, e. g. finite differences. Another limiting factor is the poor numerical characteristics of LB equations for scalar transport. As it is essential to have a minimum of numerical diffusion for the transport of the moments in the MoM in this works a Van-Leer flux splitting scheme [3] is adopted to solve the transport equations for these moments. The fluid flow is solved by the LBM which accuracy for laminar flows is widely accepted (see e.g. [4]).

\section{Particle Models}

The appropriate particle model for the presented approach under discussion must include the possibility to simulate a large number of particles of different sizes and also needs to account for their change in size. 


\subsection{Comparison of Particle Models}

Traditionally there are two approaches to model particles: The Lagrangian approach, where each particle is simulated individually by calculating its motion due to inertia and the drag force caused by the fluid. This approach can simulate the size changes in the particle population by detecting collisions between individual particles. The disadvantage of this approach is that only a limited number of particles can be treated with the available computational power. An example of a Lagrangian particle model in combination with a Lattice Boltzmann solver can be found in the papers of Redl et al [5] or Filippova et al [6].

The other approach is the Eulerian particle model. In this model the particles are represented by a density $\phi$ of particles of uniform size and properties. These particles are transported by the fluid without deviating from the streamlines using the usual transport equation:

$$
\frac{\partial \rho \phi}{\partial t}=\frac{\partial}{\partial x_{i}}\left(\rho u_{i} \phi-D \frac{\partial \phi}{\partial x_{i}}\right)=S
$$

In order for this approach to be valid the particles have to be small enough:

$$
\frac{F_{\text {drag }}}{F_{\text {inertial }}} \propto \frac{r \mu v}{r^{3} \rho v^{2}} \gg 1
$$

This approach allows an unlimited number of particles, but it can not model changes in the particle size distribution.

An approach, that is widely used together with the LBM, is the usage of a cellular automata formulation where the number of particles in each computational cell is stored. Particle transport and deposition are updated at each time-step by calculating the probability that a particle moves to a neighboring cell (using the velocities from the flow solution) and moving the corresponding number of particles. These models allow for a much larger number of particles than the Lagrangian particle model, but they assume a homogeneous particle population and they don't take particle-particle interactions into account. They have been successfully used for the calculation of snow and sand transport ([7], [8]).

\subsection{Method of Moments (MoM)}

In order to take account for the change in particle size distribution, particles are separated into classes of mass $m_{i}=i \Delta m$ where the particle class $i$ contains $N_{i}$ particles. The evolution of these particle classes can be described by the Smoluchowski model [9]:

$$
\begin{aligned}
\frac{d N_{1}}{d t} & =-\sum_{j=1}^{\infty} \beta_{1, j} N_{1} N_{j} \\
\frac{d N_{i}}{d t} & =\frac{1}{2} \sum_{j=1}^{i-1} \beta_{1, i-j} N_{j} N_{i-j}-\sum_{j=1}^{\infty} \beta_{i, j} N_{i} N_{j} \\
i & =2, \ldots, \infty
\end{aligned}
$$


with the collision operator $\beta_{i, j}$ describing the probability that two particles from the particle classes $i$ and $j$ coagulate. To simulate the complete evolution of the particle population, all these equations would have to be solved simultaneously which of course is not feasible for non-trivial PDFs.

An alternative approach is the Method of Moments (MoM). The MoM is based on the moments of the particle population:

$$
M_{r}=\sum_{i} m_{i}^{r} N_{i}
$$

Moment $M_{0}$ equals the total number of particles, moment $M_{1}$ is equivalent to the total mass of the particles. The higher order moments correspond to statistical measures of the PDF and consequently its shape.

Knowing all the moments $M_{r}$ is equivalent to knowing the exact size distribution of the particles. However, to reconstruct an approximation of the PDF only a small number of moments is needed (according to Frenklach [10] a number of 6 moments is sufficient for most applications).

The changes of the moments can be calculated from the changes of the particle classes (8):

$$
\begin{aligned}
& S_{0}=\frac{d M_{0}}{d t}=-\frac{1}{2} \sum_{i=1}^{\infty} \sum_{j=1}^{\infty} \beta_{i, j} N_{i} N_{j} \\
& S_{1}=\frac{d M_{1}}{d t}=0 \\
& S_{2}=\frac{d M_{2}}{d t}=\sum_{i=1}^{\infty} \sum_{j=1}^{\infty} i j \beta_{i, j} N_{i} N_{j} \\
& S_{3}=\frac{d M_{3}}{d t}=3 \sum_{i=1}^{\infty} \sum_{j=1}^{\infty} i j^{2} \beta_{i, j} N_{i} N_{j} \ldots
\end{aligned}
$$

For specific collision terms $\beta_{i, j}$ (for an example see [11]) the sums in these formulas can be reduced to expressions that involve only a finite number of terms that are expressed by moments $M_{r}$ (where $r$ isn't necessarily an integer number). These moments can be approximated from the known moments by interpolation.

The transport of the PDF is implemented by the usual transport equation (6) using the time evolutions of the moments (11) - (14) as source terms:

$$
\frac{\partial \rho M_{r}}{\partial t}=\frac{\partial}{\partial x_{i}}\left(\rho u_{i} M_{r}-D \frac{\partial M_{r}}{\partial x_{i}}\right)=S_{r}
$$

Equation (15) is the transport equation for the moment $M_{r}$. The source term $S_{r}$ is the source term as calculated in equations (11)-114). In the general case for the calculation of these source terms the values of the other moments are needed (coupling the calculation of the moments).

Solving equation (15) for the moments is equivalent to solving (6) for each particle class: each particle with a mass $m_{i}$ carries a contribution to $M_{r}$ of $m_{i}^{r}$. All $N_{i}$ particles in class $i$ have a moment $N_{i} m_{i}^{r}$ for which we could solve (6). If these equations are added for all particle classes and we use (10), we get to (15). 


\section{Implementation}

The Method of Moments has been integrated into the existing LBM solver. Due to the explicit nature of the LBM the calculation of the flow solution and the particle transport can be easily decoupled: at each time-step the new solution of the Navier-Stokes equations is calculated by the LBM. Then the transport equations (15) for the moments are solved using that flow solution.

Then the source terms $S_{r}$ are calculated from equations (11) - (14). For general collision terms $\beta_{i, j}$ fractional moments $M_{f}$ have to be used, which are interpolated from the (known) integer moments. Because there are several orders of magnitude between the moments, which leads to numerical instabilities in the interpolation, the interpolation is performed with the logarithms of the moments making the computation of the sources much stabler (this is possible because the moments (10) are positive).

In addition to the source terms from particle-particle interactions, in the computational cells next to walls, a source term for the particle deposition on the walls is calculated (these are obviously the only source terms $S_{1}$ for the mass density $M_{1}$ ). For each computational cell a separate variable $\rho_{\text {dep }}$ for the density of the deposited soot is introduced. The equation

$$
\frac{\partial \rho_{d e p}}{\partial t}=-S_{1}
$$

for the deposited soot is solved. As soon as $\rho_{\text {dep }}$ reaches a critical density $\rho_{\text {full }}$ (which is known from weight measurements of deposited soot) the computational cell is marked as full. It is then treated like a wall boundary cell by the LBM and soot can deposit in the neighboring cells.

An alternate approach is to treat the deposited soot as a permeable medium and allow the flow of air through through the soot modified by the DarcyForchheimer term. This method also allows for the treatment of partially filled cells and therefor a more accurate flow solution. The disadvantage is that the additional source term for the Navier-Stokes equations degrades the stability of the LBM.

The necessity to store seven additional scalars (6 moments and the deposition density) per volume cell increases the memory consumption of the simulation moderately. More significant is the increase in CPU-time, as the calculation of the particle transport and the source terms have to be performed at every timestep and are computationally intensive.

\section{Results}

Two simulation results are shown to illustrate the capabilities of the Method of Moments: the first simulation is a simple case that illustrates the effect of the particle coagulation. The second case shows particle flow in a more complex geometry. 


\subsection{Deposition in a Channel}

The first simulation shows laminar flow through a channel at a constant pressure gradient. Soot particles are inserted at the left end of the channel, and deposit eventually on the channel walls and on the rectangular obstacle located at the right end of the channel. The results are shown in figure 1]. The obstacles are shown in light grey, the deposited soot is shown in black. The values of the moments are depicted by isolines.

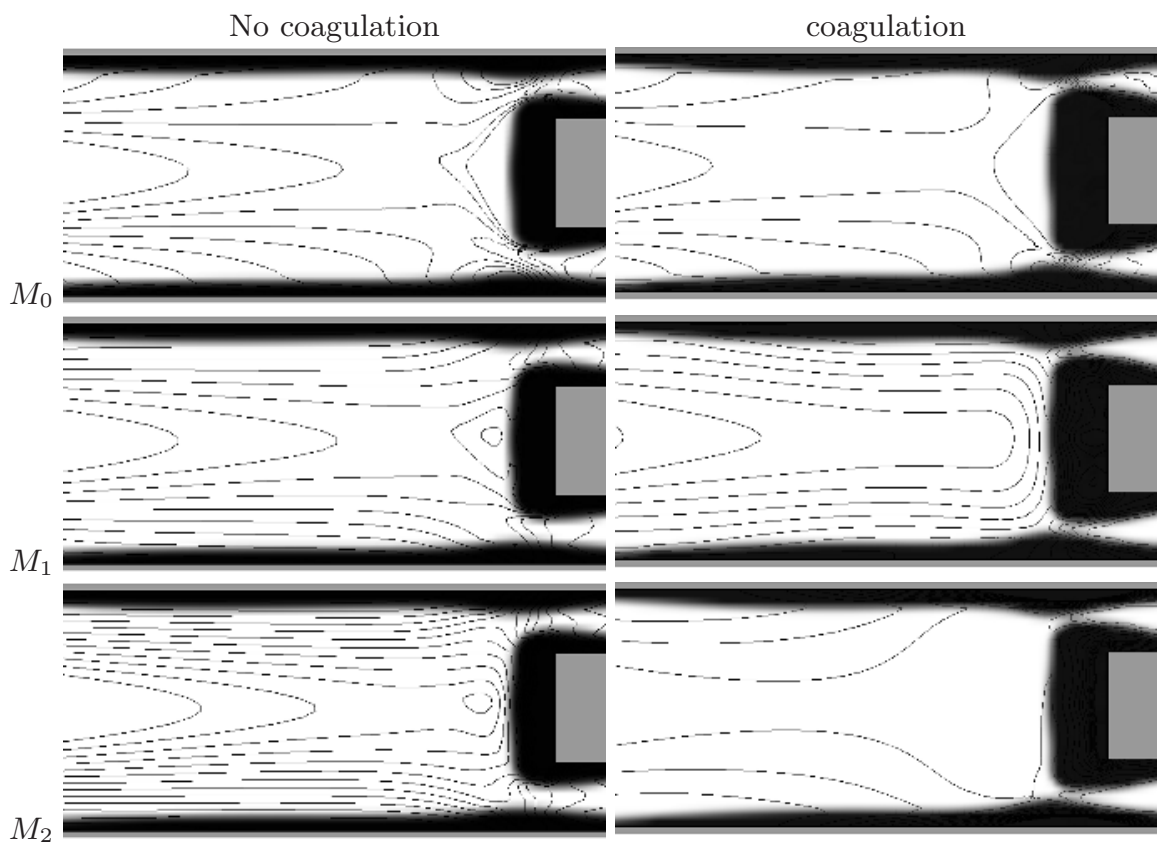

Fig. 1. Soot deposition in a channel with obstacle. The same simulation is shown with and without coagulation

In the first column the simulation without coagulation is shown. The second column shows the same simulation, but the PDF changes due to coagulation. For both simulations the result at the same time is given.

The images in the first line show the particle density, $M_{0}$. Coagulation causes a reduction in particle density in flow direction. The second line shows the material density $M_{1}$, which is nearly the same in both cases (differences near the obstacles are due to the different flow fields caused by the different soot depositions). The last line shows the moment $M_{2}$ which corresponds to the width of the PDF which is much smaller in the coagulation case.

The amount of deposited soot is larger in the case of coagulation, because the larger particles tend to deposit.more easily. 


\subsection{Deposition on a Pore Structure}

The pictures in figure 2 show fluid-particle flow through a more realistic structure, reconstructed from existing porous material. The average pore radius is 0.1 millimeters.

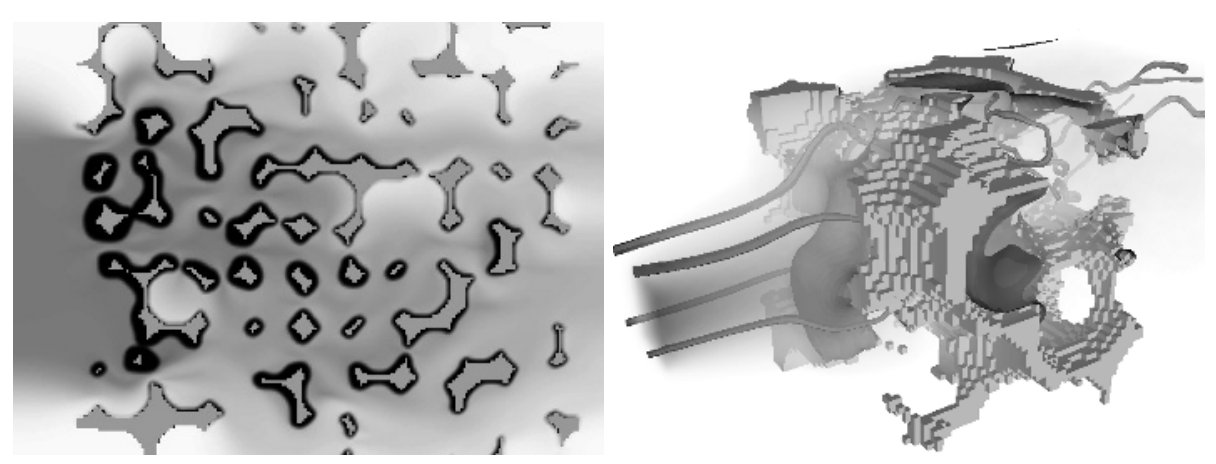

Fig. 2. Deposition in porous material. The left picture shows a two-dimensional reconstruction of a pore with deposited soot and soot density. The right picture shows a three-dimensional simulation of soot deposition on a pore structure. Stream line of the velocity, soot density in the air and soot deposited on the pore are visible

The left picture in figure 2 was obtained from a two dimensional simulation. The pore itself is shown in light gray, deposited soot in black. Flow through the pore structure goes from the left to the right and is driven by a constant pressure difference, that was applied at the boundaries. A soot distribution is inserted at the inlet. The density $M_{1}$ of the soot in the air is shown in shades of gray.

It can be clearly seen, that the density of the soot in the air drops due to the deposition of the soot on the pore structure.

The deposited soot changes the flow distribution through the pore and therefore the permeability of the filter and the filtration efficiency.

The picture on the right of figure 2 is taken from a 3D simulation of a similar pore. The pore walls are represented by the the cubes. The air flows through the pore structure from the left to the right (four "stream tubes" depict the fluid velocity). Soot is inserted at a small fraction of the inflow (soot density is shown by the gray fog). Soot deposition on the pore is shown by the dark gray isosurfaces.

\section{Conclusion}

The Method of Moments for soot particle tracking and deposition is successfully combined with the Lattice Boltzmann Method. Particle coagulation and deposition can be easily implemented by this approach. 
Application of the suggested approach has been shown for two cases: Soot deposition in simple geometries and realistic porous structures.

The combination of these two methods makes it possible to perform simulations in a wide range of applications, where complex geometries and changing particle density functions are encountered.

\section{References}

1. Chen, S., Doolen, G.D.: Lattice boltzmann method for fluid flos. Ann. Rev. Fluid Mech. (1998)

2. Yu, D., Mei, R., Luo, L.S., Shyy, W.: Viscous flow computations with the method of lattice boltzmann equation. Progress in Aerospace Sciences (2003)

3. Leer, B.V.: Toward the ultimate convervative difference scheme v. Journal of Computational Physics (1979)

4. Luo, L.S.: The lattice gas and lattice boltzmann methods: Past, present and future. In: Proceedings "International Conference on Applied Computational Fluid Dynamics", Beijing, China. (2000)

5. Redl, C., Wassermayr, C., Leixnering, J.: On the numerical simulation in foam filter design for diesel exhaus gas systems. In: Proceedings "International Congress for Engine Combustion Process", Munich, Germany. (2003)

6. Filippova, O., Hänel, D.: Lattice-boltzmann simlation of gas-particle flow in filters. Computers \& Fluids (1997)

7. Chopard, B., Masselot, A., Dupuis, A.: A lattice gas model for erosion and particle transport in a fluid. Computer Physics Communications (2000)

8. Dupuis, A., Chopard, B.: Lattice gas modeling of scour formation under submarine pipelines. Journal of Computational Physics (2002)

9. Smoluchowski, M.: Versuch einer mathematischen theorie der koagulationskinetik kolloider lösungen. Zeitschrift für physikalische Chemie (1917)

10. Frenklach, M.: Method of moments with interpolative closure. Chemical Engineering Science (2002)

11. Kazakov, A., Frenklach, M.: Dynamic modeling of soot particle coagulation and aggregation: Implementation with the method of moments and application to highpressure laminar premixed flames. Combustion and Flame (1998)

12. McGraw, R., Wright, D.L.: Chemically resolvedaerosol dynamics for internal mixtures by the quadrature method of moments. Journal of Aerosol Science (2003)

13. Wu, J., Hu, B.X., Zhang, D., Shirley, C.: A three-dimensional numerical method of moments for groundwater flow and solute transport in a nonstationary conductivity field. Advances in Water Resources (2003) 